\title{
Multiplex Molecular Stool Testing Rarely Impacts Antimicrobial Treatment Decisions More Than Three Days After Admission
}

\author{
Abdul Aleem ${ }^{1}$, Gabriela Firak ${ }^{2}$, Amy K. Slenker ${ }^{3}$ \\ 1. Gastroenterology and Hepatology, Lehigh Valley Health Network, Allentown, USA 2. Internal Medicine, Tower \\ Health-Reading Hospital, Reading, USA 3. Infectious Diseases, Lehigh Valley Health Network, Allentown, USA
}

Corresponding author: Amy K. Slenker, amy_k.slenker@lvhn.org

\section{Abstract \\ Background}

Acute diarrheal illness in the United States is a significant cause of healthcare utilization and hospitalizations. For patients who develop diarrhea while hospitalized, testing for pathogens other than Clostridium difficile ( $C$. difficile) using conventional stool testing is low yield. Newer testing modalities for infectious diarrhea such as the multiplex molecular stool testing provide an improved detection rate and a faster turn-around time compared to conventional stool testing.

\section{Methods}

We retrospectively examined the use of a multiplex molecular stool test at our institution for all hospital encounters over a two-year period to determine which organisms were identified $\leqslant 3$ days and $>3$ days after admission.

\section{Results}

A total of 2032 patients underwent multiplex molecular stool testing during the study period, with 1698 (83.6\%) performed $\leqslant 3$ days and $334(16.3 \%)>3$ days after admission. An enteric non-C. difficile pathogen was identified more frequently when patients were tested $\leqslant 3$ days after admission $(350,20.6 \%)$ as compared to $>3$ days after admission $(38,11.4 \%, p<0.0001)$. Excluding coinfections, $C$. difficile was identified more frequently when patients were tested $>3$ days after admission (64, 20.3\%) versus another organism (30, $9.0 \%)(p<0.0001)$. Of those patients with a non- $C$. difficile pathogen identified $>3$ days after admission, a bacterial pathogen amenable to treatment was only identified in $6 \%$ (21) of patients.

\section{Conclusion}

Multiplex molecular stool testing for patients tested $>3$ days after admission is a low yield of information that could guide antimicrobial treatment decisions, and $C$. difficile testing is more useful in this clinical

Review began 04/20/2021 Review ended 04/27/2021 Published 04/30/2021

\section{๑) Copyright 2021}

Aleem et al. This is an open access article distributed under the terms of the Creative Commons Attribution License CC-BY 4.0., which permits unrestricted use, distribution, and reproduction in any medium, provided the original author and source are credited.
Categories: Internal Medicine, Gastroenterology, Infectious Disease

Keywords: molecular stool testing, c. difficile, diarrhea, 3-day rule, antimicrobial treatment decisions

\section{Introduction}

Acute diarrheal illness is a significant cause of morbidity and mortality worldwide, with the most common infectious etiologies being Norovirus, Campylobacter spp., and non-typhoidal Salmonella infections [1]. In resource-rich countries, such as the United States, diarrhea illness is a rare cause of death but is a significant source of outpatient care utilization and hospitalizations [2]. In the US, diagnostic testing for communityonset diarrhea should be performed in patients with fever, bloody or mucoid stools, severe symptoms, or an immunocompromised state who would benefit from antimicrobial therapy [3].

Conversely, for patients who develop diarrhea while hospitalized, testing for infectious etiologies via stool culture for enteric pathogens is low value, as well as costly [4, 5]. Prior studies with conventional stool testing methods have shown that in patients who develop diarrhea while hospitalized, testing for pathogens other than Clostridium difficile (C. difficile) is low yield when performed $>3$ days after admission [6], which has led to the adoption of the "3-day rule" by many laboratories.

Conventional testing methods are increasingly being replaced by multiplex molecular stool testing, which provides an improved detection rate for multiple pathogens simultaneously with a high sensitivity and a rapid turnaround time [7-9]. However, it is not clear if the "3-day rule" should be applied to this new test. Prior studies on this topic revealed the percent positivity rates by molecular testing for stool samples 
Our institution began using multiplex molecular stool testing in place of routine stool culture for the diagnosis of suspected infectious diarrhea on January 5, 2015. This test identifies 22 different bacterial, viral, and parasitic stool pathogens, including $C$. difficile. In this study, we retrospectively examined the use of a multiplex molecular stool test for all hospital encounters over a two-year period to determine which organisms were identified in patients who are tested after being hospitalized $\leqslant 3$ days and $>3$ days. We also aimed to evaluate if prior guidance regarding a " 3 -day rule" is applicable in the molecular testing age.

\section{Materials And Methods}

This was a retrospective chart review study of all adult patients 18 years or older who presented to Lehigh Valley Health Network (LVHN) Cedar Crest or Muhlenberg campuses from January 5, 2015, to January 4, 2017, who had a multiplex molecular stool test performed during their hospital encounter. The patient's charts were reviewed to obtain age, gender, admission date, stool testing date, and the result of stool testing. Repeat testing during the same hospital admission was not included. Patients with indeterminate results were excluded.

The specimens were received by the laboratory in Cary-Blair enteric transport medium and were tested via the FilmArray Gastrointestinal (GI) Panel (BioFire Inc., Salt Lake City, UT, USA) according to the package insert. The FilmArray ${ }^{\circledR}$ GI Panel looks for the following pathogens: Campylobacter (jejuni, coli, and upsaliensis), Clostridium difficile (Toxin A/B), Plesiomonas shigelloides, Salmonella, Vibrio (parahaemolyticus, vulnificus, and cholerae), Yersinia enterocolitica, Enteroaggregative Escherichia coli (EAEC), Enteropathogenic Escherichia coli (EPEC), Enterotoxigenic Escherichia coli (ETEC), Shiga-like toxinproducing Escherichia coli (STEC), Escherichia coli O157, Shigella/Enteroinvasive Escherichia coli (EIEC), Cryptosporidium, Cyclospora cayetanensis, Entamoeba histolytica, Giardia lamblia, Adenovirus F 40/41, Astrovirus, Norovirus GI/GII, Rotavirus A, and Sapovirus (I, II, IV, and V).

The primary endpoint was to determine if there is a significant difference in the percentage of tests resulting with enteric (non-C. difficile) pathogens if the testing was performed $\leqslant 3$ or $>3$ days after hospital admission. A secondary endpoint included determining if there was a difference in the percentage of tests resulting with $C$. difficile versus other enteric, non- $C$. difficile pathogens if the testing was performed $>3$ days after hospital admission.

\section{Statistical analysis}

Continuous variables were described using the median and interquartile range (IQR), and categorical variables were described with frequency and percentage. When excluding coinfections, the Chi-Square test was used as patients could only be placed into one group. When including co-infections, McNemar's test was used as patients could have tested positive for both enteric non-C. difficile pathogens and C. difficile. All statistical analyses were conducted using SAS 9.3 (SAS Institute, Cary, NC, USA).

\section{Results}

The study sample consisted of 2032 patients, of which the median age was 64 (IQR 51-76) years, and 854 were males (42\%). The median number of days into the patient's hospital stay that the multiplex molecular stool test was performed was 1 (range 0-63) day. In total, 703 (34.6\%) patients tested positive for any pathogen: 315 (15.5\%) patients tested positive for $C$. difficile alone, 333 (16.4\%) patients tested positive for at least one pathogen other than C. difficile, and 55 (2.7\%) patients tested positive for both $C$. difficile and at least one other pathogen (Table 1). 


\section{Cureus}

\begin{tabular}{|c|c|c|c|}
\hline Characteristic & Total $(n=2032)$ & $\leq 3$ Days $(n=1698)$ & $>3$ Days $(n=334)$ \\
\hline Age, years median (IQR) & $64(51-76)$ & $63(49-75)$ & $67(56-77)$ \\
\hline \multicolumn{4}{|l|}{ Gender } \\
\hline Male & $854(42.0)$ & 699 (41.2) & $155(46.4)$ \\
\hline Female & $1178(58.0)$ & $999(58.8)$ & $179(53.6)$ \\
\hline Day after admission test done median (IQR) & $1(0-2)$ & $1(0-1)$ & $8(5-12)$ \\
\hline \multicolumn{4}{|l|}{ Positive test result } \\
\hline Yes & $703(34.6)$ & $601(35.4)$ & $102(30.5)$ \\
\hline No & $1329(65.4)$ & 1097 (64.6) & $232(69.5)$ \\
\hline \multicolumn{4}{|l|}{ Infection status } \\
\hline No infection detected & $1329(65.4)$ & $1097(64.6)$ & $232(69.5)$ \\
\hline Infected with C. difficile only & 315 (15.5) & $251(14.8)$ & 64 (19.2) \\
\hline Infected with other (non-C. difficile) pathogen only & $333(16.4)$ & $303(17.8)$ & $30(9.0)$ \\
\hline Infected with both C. difficile and other pathog & $55(2.7)$ & $47(2.8)$ & $8(2.4)$ \\
\hline
\end{tabular}

\section{TABLE 1: Patient Demographics and Test Results}

Data are $n(\%)$ unless otherwise stated; percentages might not add up to $100 \%$ due to rounding.

C. difficile, Clostridium difficile

The frequency and percentage of patients who tested positive for all pathogens included in the multiplex molecular stool assay, as well as the breakdown of the organism identified as compared to the day of testing $(\leqslant 3$ days versus $>3$ days after admission), is shown in Table 2 . An enteric, non-C. difficile pathogen was identified in 350 patients $(20.6 \%$ ) when patients were tested $\leqslant 3$ days after admission as compared to $>3$ days after admission $(38,11.4 \%, p<.0001)$ (Table 2). 


\section{Cureus}

\begin{tabular}{|c|c|c|c|}
\hline Pathogen & Total $(n=2032)$ & $\leq 3$ Days $(n=1698)$ & $>3$ Days $(n=334)$ \\
\hline Clostridium difficile (Toxin A/B) & 370 (18.2) & 298 (17.6) & $72(21.6)$ \\
\hline Total Other Pathogen (not C. difficile) & $388(19.1)$ & $350(20.6)$ & $38(11.4)$ \\
\hline Campylobacter (jejuni, coli, and upsaliensis) & $41(2.0)$ & $38(2.2)$ & $3(0.9)$ \\
\hline Plesiomonas shigelloides & $4(0.2)$ & $4(0.2)$ & 0 \\
\hline Salmonella & $17(0.8)$ & $15(0.9)$ & $2(0.6)$ \\
\hline Vibrio (parahaemolyticus, vulnificus, and cholera) & $1(0.05)$ & 0 & $1(0.3)$ \\
\hline Vibrio Cholera & $1(0.05)$ & $1(0.1)$ & 0 \\
\hline E. coli EAEC & $39(1.9)$ & $36(2.1)$ & $3(0.9)$ \\
\hline E. coli EPEC & $116(5.7)$ & $102(6.0)$ & $14(4.2)$ \\
\hline E. coli ETEC & $11(0.5)$ & $9(0.5)$ & $2(0.6)$ \\
\hline E. coli STEC & $10(0.5)$ & $10(0.6)$ & 0 \\
\hline E. coli 0157 & $10(0.5)$ & $8(0.5)$ & $2(0.6)$ \\
\hline E. coli EIEC & $10(0.5)$ & $10(0.6)$ & 0 \\
\hline Cryptosporidium & $10(0.5)$ & $10(0.6)$ & 0 \\
\hline Cyclospora cayetanesis & 0 & - & - \\
\hline Entamoeba histolytica & 0 & - & - \\
\hline Giardia lamblia & $6(0.3)$ & $5(0.3)$ & $1(0.3)$ \\
\hline Adenovirus F 40/41 & $5(0.3)$ & $4(0.2)$ & $1(0.3)$ \\
\hline Astrovirus & $8(0.4)$ & $8(0.5)$ & 0 \\
\hline Norovirus GI/GII & $124(6.1)$ & $115(6.8)$ & $9(2.7)$ \\
\hline Rotavirus A & $7(0.3)$ & $7(0.4)$ & 0 \\
\hline Sapovirus (I, II, IV, and V) & $17(0.8)$ & $17(1.0)$ & 0 \\
\hline Yersinia enterocolitica & $11(0.5)$ & $10(0.6)$ & $1\left(0.3^{3}>3\right)$ \\
\hline
\end{tabular}

\section{TABLE 2: Positive Test Results by Pathogen and Day of Testing After Admission}

Data are $n(\%)$ unless otherwise stated; percentages might not add to $100 \%$ due to rounding; table includes coinfections.

EAEC, enteroaggregative; EPEC, enteropathogenic; ETEC, enterotoxigenic; STEC, Shiga-like toxin-producing; EIEC, Shigella/enteroinvasive; E. Coli, Escherichia coli; C. difficile: Clostridium difficile

Clostridium difficile was the most prevalent individual organism identified in patients tested both $\leqslant 3$ days and $>3$ days after admission $(298,17.6 \%)$ versus $(72,21.6 \%)$. After excluding coinfections, patients were more likely to have $C$. difficile diagnosed if tested $>3$ days as compared to $\leqslant 3$ days after admission $(64,19.6 \%$ versus $251,15.2 \%, p=0.0459$ ).

After excluding coinfections, in patients who had a multiplex molecular stool test performed $>3$ days after admission, C. difficile was identified in 64 patients $(20.3 \%)$ versus other organisms $(30,9.0 \%)(p<0.0001)$ (Table 3). Of those, 30 patients tested $>3$ days after admission, the following pathogens were identified: Adenovirus F40/41 (1), Campylobacter Spp. (2), Escherichia coli (EAEC) (3), Escherichia coli (EPEC) (10), Escherichia coli (ETEC) (2), Giardia lamblia (1), Norovirus GI/GII (8), Salmonella sp. (1), Vibrio sp. (1), Yersinia enterocolitica (1). 


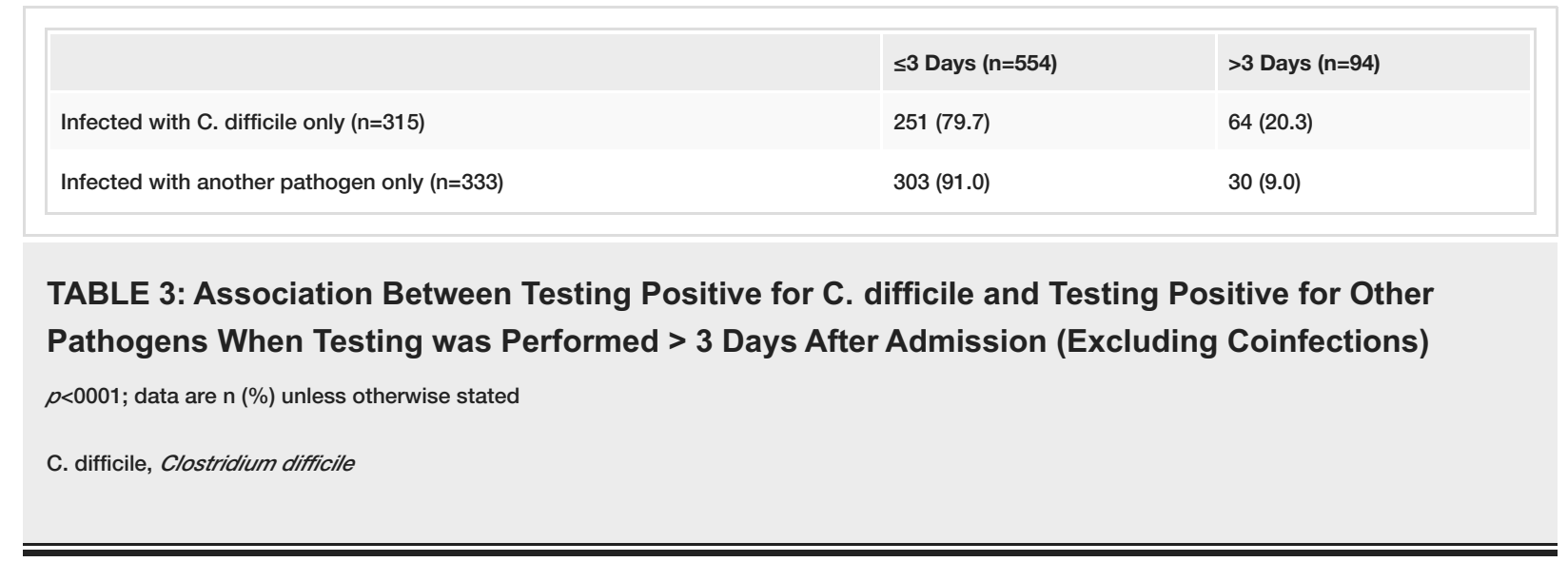

\section{Discussion}

This retrospective study reviewed the use of multiplex molecular stool testing performed $\leqslant 3$ and $>3$ days after admission at a large academic institution over a two-year period. Clinical decision support was not instituted with the transition from conventional stool testing to multiplex molecular stool testing and subsequently, it was noted that providers were using this test indiscriminately for all hospitalized patients with concern for infectious diarrhea.

Most patients had stool testing performed early in the admission (median 1, range 0-63); however, 334 (16.3\%) of patients had multiplex molecular stool testing performed $>3$ days after admission. For patients tested $>3$ days after admission, testing was negative in 232 (69.5\%), consistent with previous findings that nosocomial diarrhea is often noninfectious in etiology [12].

Patients were more likely to be diagnosed with an enteric non- $C$. difficile pathogen if tested $\leqslant 3$ days after admission (350, 20.6\%) as compared to $>3$ days after admission $(38,11.4 \%, p<.0001)$. This is consistent with previous data showing that stool testing for infectious pathogens is higher yield earlier in admission. Importantly, after excluding coinfections, patients who had multiplex molecular stool test performed $>3$ days after admission were more likely to have $C$. difficile identified (64, 20.3\%) versus another organism (30, $9.0 \%$ ) (Table $1, p<0.0001$ ). After a case review of those 30 patients tested $>3$ days after admission who a pathogen other than $C$. difficile identified, only 21 (6\%) were bacterial causes potential amenable to treatment, and of those, only 7 (2\%) patients received treatment.

This strength of this study is that it is a large study reviewing the real-life application of a novel testing modality. This study provides actionable data for other institutions transitioning to multiplex molecular stool testing by supporting the implementation of clinical decision support for a "3-day rule" to discourage molecular stool testing $>3$ days after admission. The limitations of this study include its retrospective nature and that it was performed at a single healthcare institution.

\section{Conclusions}

Hospitalized patients with diarrhea tested $>3$ days after admission using multiplex molecular stool testing were more likely to have $C$. difficile identified versus another enteric organism. Clinical decision support is advised to guide providers to preferentially perform $C$. difficile testing for patients hospitalized $>3$ days as opposed to multiplex molecular stool testing.

\section{Additional Information}

\section{Disclosures}

Human subjects: Consent was obtained or waived by all participants in this study. Lehigh Valley Health Network IRB issued approval STUDY00000183. Dear Amy Slenker: On 10/24/2018, the IRB reviewed the following submission: Type of Review: Initial Study Title of Study: Inappropriate Use of a Multiplex Stool PCR Assay in Hospitalized Patients Who Develop Diarrhea at a Large Academic Institution Investigator: Amy Slenker IRB ID: STUDY00000183 Funding: None Grant ID: None IND, IDE, or HDE: None Documents Reviewed: • Inappropriate Use Stool PCR Variable Codebook 10-2-18 final.docx, Category: Other; • Investigator-Initiated, Amy Slenker, Version 3, October 2 2018.docx, Category: IRB Protocol; • InvestigatorInitiated, Amy Slenker, Version 2, October 1 2018.docx, Category: IRB Protocol; • Investigator-Initiated, Amy Slenker, Version 1, May 16 2017.docx, Category: IRB Protocol The IRB approved the study from 10/24/2018 to 10/23/2019 inclusive. Before 10/23/2019 or within 30 days of study closure, whichever is earlier, you are to submit a continuing review with required explanations. You can submit a continuing review by navigating to the active study and clicking Create Modification / CR. If continuing review approval is not granted on or before 10/23/2019, approval of this study expires after that date. A study closure is also submitted using the Create Modification / CR activity. In conducting this study, you are required to follow the requirements listed 
in the Investigator Manual (HRP-103), which can be found by navigating to the IRB Library within the IRB system. Sincerely, Leroy Kromis, PharmD IRB Chair. Animal subjects: All authors have confirmed that this study did not involve animal subjects or tissue. Conflicts of interest: In compliance with the ICMJE uniform disclosure form, all authors declare the following: Payment/services info: All authors have declared that no financial support was received from any organization for the submitted work. Financial relationships: All authors have declared that they have no financial relationships at present or within the previous three years with any organizations that might have an interest in the submitted work. Other relationships: All authors have declared that there are no other relationships or activities that could appear to have influenced the submitted work.

\section{Acknowledgements}

The authors would like to thank the following people for their assistance: Daniel Lindao, MS, M (ASCP), Georgia Colasante, MS, MT (ASCP), SM (NRM) from Health Network Laboratories, and Jennifer Macfarlan, MPH from the Lehigh Valley Health Network Office of Research and Innovation.

\section{References}

1. Kirk MD, Pires SM, Black RE, et al.: World Health Organization estimates of the global and regional disease burden of 22 foodborne bacterial, protozoal, and viral diseases, 2010: a data synthesis. PLoS Med. 2015, 12:e1001921. 10.1371/journal.pmed.1001921

2. Scallan E, Hoekstra RM, Angulo FJ, et al.: Foodborne illness acquired in the United States--major pathogens . Emerg Infect Dis. 2011, 17:7-15. 10.3201/eid1701.p11101

3. Shane AL, Mody RK, Crump JA, et al.: 2017 Infectious Diseases Society of America clinical practice guidelines for the diagnosis and management of infectious diarrhea. Clin Infect Dis. 2017, 65:e45-80. 10.1093/cid/cix669

4. McFarland LV: Epidemiology of infectious and iatrogenic nosocomial diarrhea in a cohort of general medicine patients. Am J Infect Control. 1995, 23:295-305. 10.1016/0196-6553(95)90060-8

5. Samore MH, DeGirolami PC, Tlucko A, Lichtenberg DA, Melvin ZA, Karchmer AW: Clostridium difficile colonization and diarrhea at a tertiary care hospital. Clin Infect Dis. 1994, 18:181-7. 10.1093/clinids/18.2.181

6. Rohner P, Pittet D, Pepey B, Nije-Kinge T, Auckenthaler R: Etiological agents of infectious diarrhea: implications for requests for microbial culture. J Clin Microbiol. 1997, 35:1427-32. 10.1128/JCM.35.6.14271432.1997

7. Zhang H, Morrison S, Tang YW: Multiplex polymerase chain reaction tests for detection of pathogens associated with gastroenteritis. Clin Lab Med. 2015, 35:461-86. 10.1016/j.cll.2015.02.006

8. Buss SN, Leber A, Chapin K, et al.: Multicenter evaluation of the BioFire FilmArray gastrointestinal panel for etiologic diagnosis of infectious gastroenteritis. J Clin Microbiol. 2015, 53:915-25. 10.1128/JCM.02674-14

9. Halligan E, Edgeworth J, Bisnauthsing K, et al.: Multiplex molecular testing for management of infectious gastroenteritis in a hospital setting: a comparative diagnostic and clinical utility study. Clin Microbiol Infect. 2014, 20:0460-7. 10.1111/1469-0691.12476

10. Beal SG, Velez L, Tremblay EE, Toffel S, Rand KH: The "3-day rule" for stool tests may not apply when using PCR panels. J Clin Microbiol. 2018, 56: 10.1128/JCM.02012-17

11. Hitchcock MM, Gomez CA, Banaei N: Low yield of FilmArray GI panel in hospitalized patients with diarrhea: an opportunity for diagnostic stewardship intervention. J Clin Microbiol. 2018, 56: 10.1128/JCM.01558-17

12. Polage CR, Solnick JV, Cohen SH: Nosocomial diarrhea: evaluation and treatment of causes other than Clostridium difficile. Clin Infect Dis. 2012, 55:982-9. 10.1093/cid/cis551 